\title{
Intra-operative Imaging Techniques During Surgical Management of Gliomas
}

\author{
Phiroz E Tarapore, ${ }^{1}$ Edward F Chang ${ }^{2}$ and Mitchel S Berger ${ }^{3}$ \\ 1. Resident; 2. Assistant Professor of Neurological Surgery and Physiology; 3. Professor and Chairman, \\ Department of Neurological Surgery, University of California, San Francisco
}

\begin{abstract}
The goal of glioma surgery is to maximise tumour removal while preserving existing function. Intra-operative imaging techniques play an important part in achieving this goal. This article surveys those techniques and discusses the indications, advantages and drawbacks of each. Structural techniques such as intra-operative magnetic resonance imaging (MRI), ultrasound, diffusion tensor imaging and 5-aminolevulinic acid staining offer anatomical information. Functional techniques such as functional MRI, magnetoencephalography and transcranial magnetic stimulation provide information about the functionality of brain regions. When incorporated into a frameless stereotactical neuronavigation system, these modalities increase both the efficacy and safety of glioma surgery by allowing the surgeon to achieve the most extensive and safe resection possible.
\end{abstract}

\section{Keywords}

Frameless stereotactical neuronavigation, 5-aminolevulinic acid, intra-operative ultrasound, intra-operative magnetic resonance imaging, diffusion tensor imaging, magnetoencephalography, transcranial magnetic stimulation, intra-operative imaging, functional magnetic resonance imaging

Disclosure: The authors have no conflicts of interest to declare.

Received: 1 November 2011 Accepted: 10 November 2011 Citation: European Neurological Review, 2012;7(1):76-80 DOI:10.17925/ENR.2012.07.01.76 Correspondence: Phiroz E Tarapore, Department of Neurological Surgery, University of California, San Francisco, 505 Parnassus Avenue, Box 0112, San Francisco, CA 94143-0112, US. E: taraporep@neurosurg.ucsf.edu

The goal of glioma surgery is to maximise tumour resection while preventing a new post-operative neurological deficit. For both low- and high-grade gliomas, increased extent of resection correlates with improved progression-free survival as well as with overall survival. ${ }^{1-5}$ While some features of brain tumours can be visualised, in general, most aspects of infiltrative gliomas cannot be clearly seen by direct vision, thus making it difficult to evaluate when the resection is complete. Furthermore, identifying deeper structures in the trajectory of resection is critical to preserving subcortical white matter tracts and blood vessels. For these reasons, recent advances have made intra-operative imaging a cornerstone of modern glioma neurosurgery.

Neuroimaging techniques fall into two broad groups: structural and functional. Techniques such as frameless stereotaxy, intra-operative magnetic resonance imaging (MRI), ultrasound, diffusion tensor imaging (DTI), and 5-aminolevulinic acid (5-ALA) staining provide anatomical and structural information, helping to identify normal structures and tumoural regions. Functional techniques such as functional MRI (fMRI), magnetoencephalography (MEG), and transcranial magnetic stimulation (TMS) yield information about the functionality of given brain regions. Typically, functional imaging data are acquired pre-operatively and applied intra-operatively. Depending on the technique, navigational data are acquired either pre-operatively or intra-operatively and applied intra-operatively. This article discusses both categories of neuroimaging, since they both have intra-operative applications and are critical to the successful management of gliomas.

\section{Frameless Stereotaxy}

Frameless stereotactical neuronavigation systems are the mainstay of modern image-guided neurosurgery. ${ }^{6}$ Introduced in the 1980s, frameless stereotactical neuronavigation allows the surgeon to navigate in three dimensions within the anatomy of a specific patient in real time, making use of images that are acquired pre-operatively (see Figure 1). This technique depends on accurate co-registration between the patient and the scan. An infrared emitter and receiver system records the co-ordinates of each of the fiducial points on the patient's head or the head shape in 3D space with respect to a reference arc next to the head. The software then calculates the position of the patient in space and co-registers the patient to the scan. Thereafter, touching the probe anywhere on or in the patient's head will cause the neuronavigational system to display the relevant slices of the scan, with a crosshair indicating the position of the probe tip.

Frameless stereotactical neuronavigation has greatly improved the accuracy and safety of glioma surgery. It effectively allows a resection to be carried up to its safe margin. In so doing, this technique has been shown to improve the extent of resection and to reduce post-operative deficits. ${ }^{7-10}$ Additionally, it enables the surgeon to tailor craniotomies with greater accuracy, allowing for smaller exposures, shorter incisions, and reduced morbidity. ${ }^{11}$ Finally, as stated previously, frameless stereotactical neuronavigation forms the platform by which pre-operative functional imaging data may be applied intra-operatively.

Frameless stereotactic neuronavigation does have limitations, which fall into two categories overall. The first consists of poor co-registration 
Figure 1: A Frameless Stereotactical Intra-operative Navigation System Display
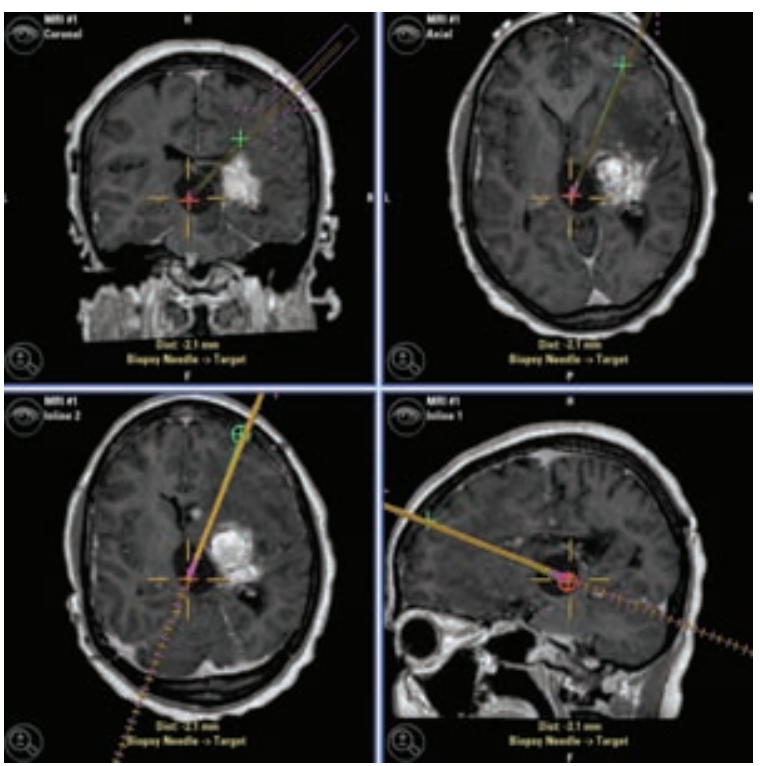

Figure 3: Low-field Intra-operative Magnetic

Resonance Imaging Scans of a Patient

Undergoing Resection of Glioma

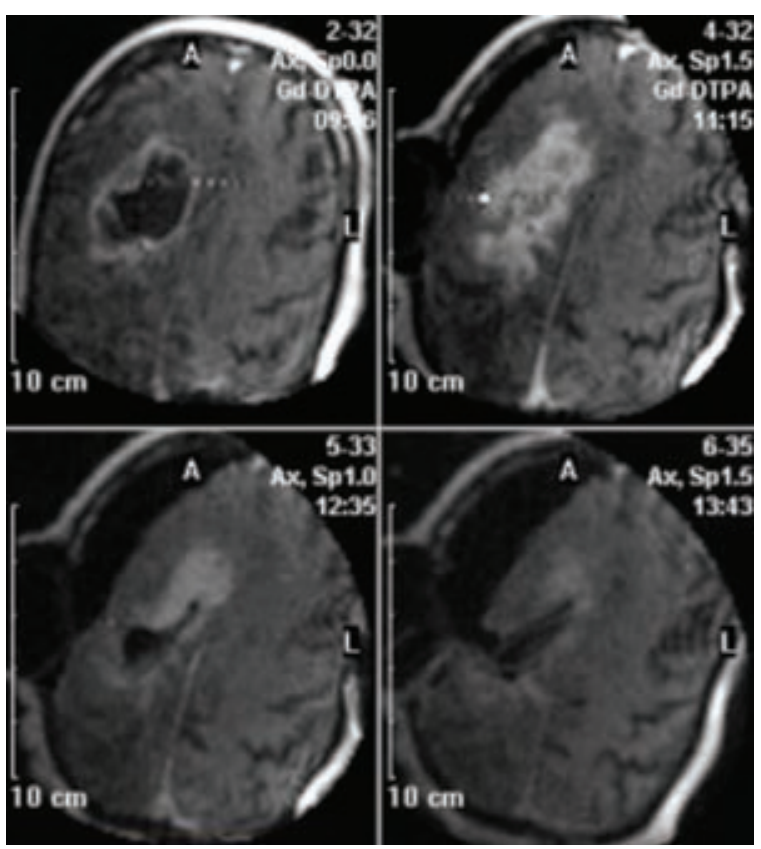

The resection cavity is evident in the right frontal region. Image courtesy of Pieter Kubben Maastricht University Medical Centre, AZ Maastricht.

between the scan and the patient, usually from non-optimal fitting of the pre-operative MRI and intra-operatively acquired landmarks. Even a few millimetres of shift in the location of the reference markers from positioning the patient can introduce problems in co-registration. Another limitation stems from intra-operative brain shift or oedema. While these limitations are of concern, newer methods such as intra-operative MRI and intra-operative ultrasound (IUS) are being developed to address these issues.

\section{Intra-operative Magnetic Resonance Imaging}

As its name suggests, intra-operative MRI refers to the acquisition of the MRI using a specialised scanner in the operating room itself. ${ }^{12}$
Figure 2: High-field Pre-operative, Intra-operative and Post-operative Magnetic Resonance Imaging Scans of Patients Undergoing Awake Craniotomy with Language Mapping

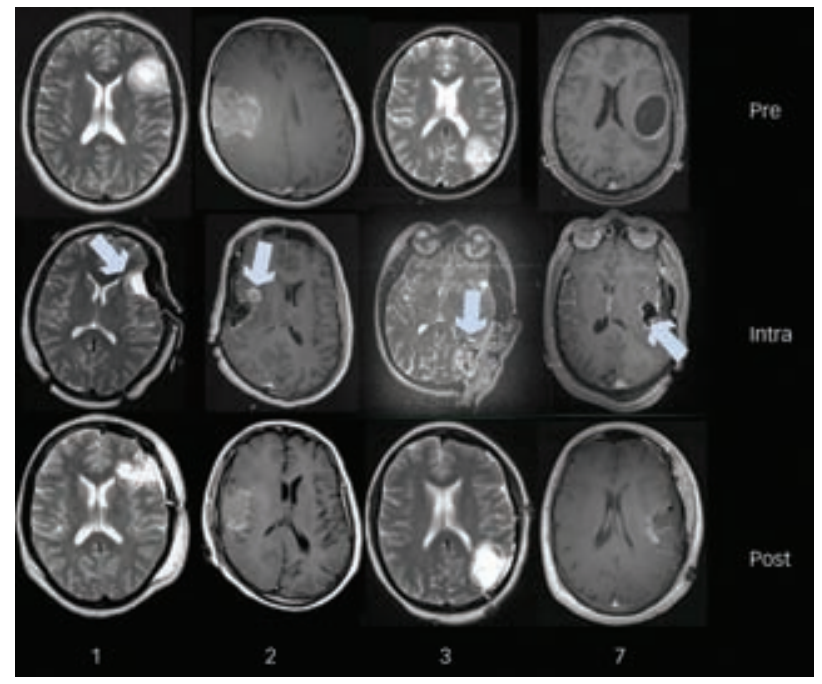

The blue arrows depict additional areas of tumour that were identified and resected after intra-operative magnetic resonance imaging. Source: Leuthardt et al., 2011. ${ }^{13}$

\section{Figure 4: Intra-operative Ultrasound of a Cystic Glioma}
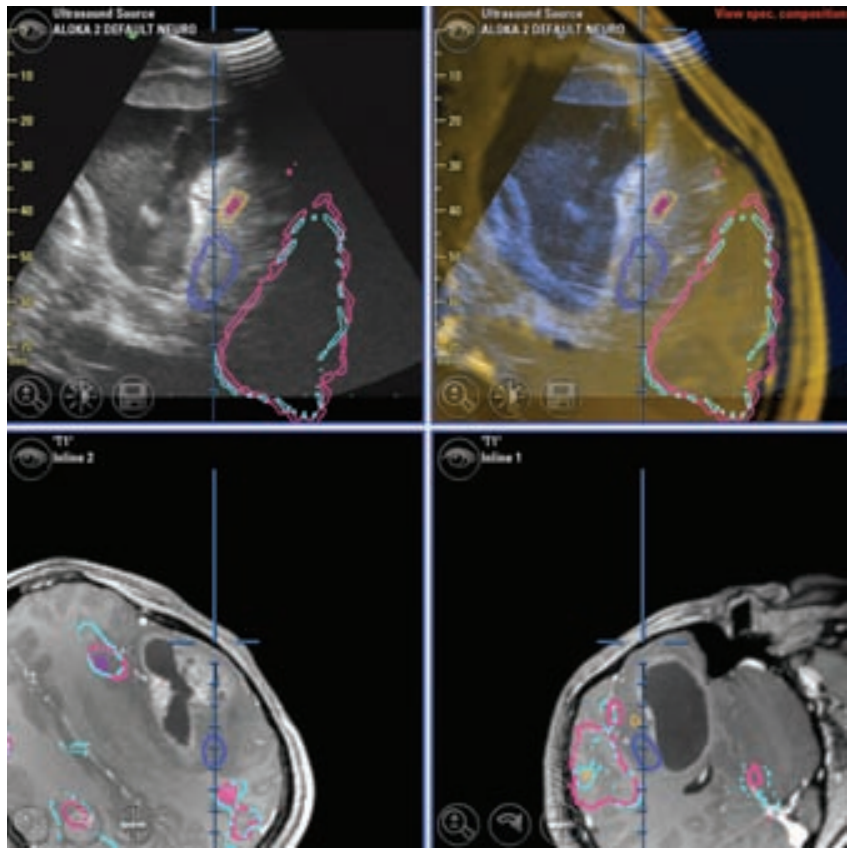

The ultrasound image is co-registered to a magnetic resonance imaging scan using a frameless stereotactical navigation system.

High-field intra-operative MRI usually functions at $1.5 \mathrm{~T}$ or greater and yields high-resolution scans (see Figure 2). These units are bigger and often require that the surgical suite be designed for rapid, easy transport of the patient to the scanner for image acquisition, such as with a motorised table that is continuous with the bore of the scanner. Low-field scanners usually function at or below $0.5 \mathrm{~T}$ and are less expensive than the high-field scanners (see Figure 3). Although these images are of lower resolution, the machines are smaller and may thus be moved over a stationary patient. ${ }^{13}$

Intra-operative MRI can confer great advantages in glioma surgery. ${ }^{14,15}$ First, it allows for the most accurate navigational co-registration 
Figure 5: Resection of a Glioma that Has Been Labelled with 5-Aminolevulinic Acid

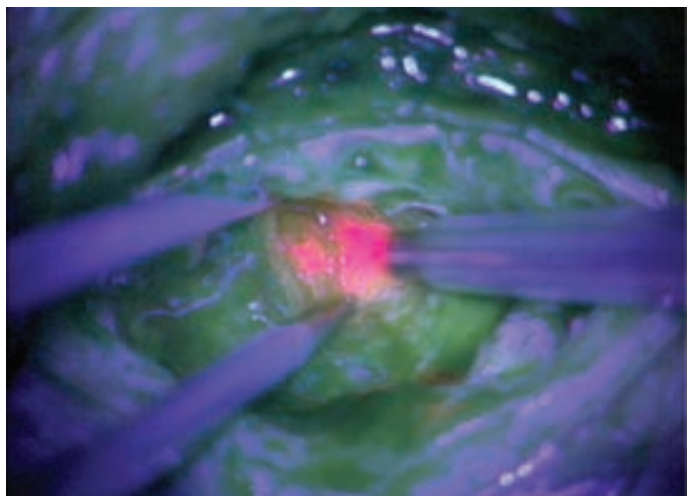

Under the fluorescent lighting of the operating microscope, the malignant tissue is centrally located and glowing with an apparent deep pink colour.

Figure 7: Diffusion Tensor Imaging Tractography in a Patient with Glioma
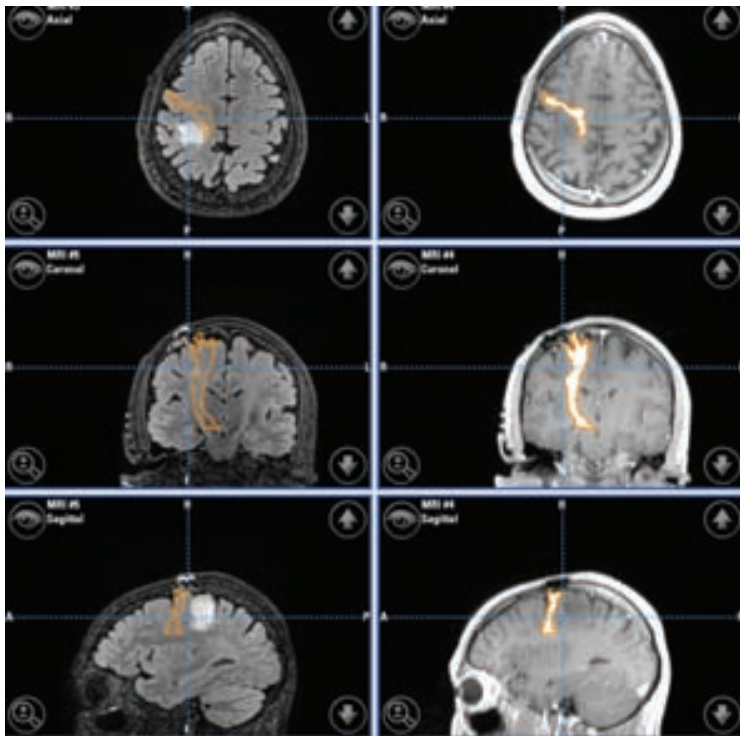

The regions highlighted in orange correspond to descending corticospinal fibre tracts. The tractography has been integrated with a neuronavigational system for intra-operative use.

because the scan is performed after the patient has been positioned for surgery. Fiducial markers are unnecessary and only the external reference marker is required. Second, intra-operative MRI allows the surgeon to evaluate the extent of resection using a gadolinium contrast agent if desired and to continue to remove any residual tumour if found. In traditional glioma surgery, this interim assessment is impossible; the surgeon must finish the case and the post-operative scan is completed the next day. Third, if brain shift occurs during surgery, an interim intra-operative scan can provide updated imaging data that accurately reflect the current anatomy.

Intra-operative MRI has been shown to improve surgical outcomes. ${ }^{14-16}$ In comparison with traditional methods, it improves the extent of resection $^{17-19}$ in both low-grade ${ }^{2,20}$ and high-grade ${ }^{16,21}$ gliomas and has been shown to reduce the size of residual tumour in the case of subtotal resection..$^{22}$ In a randomised controlled trial, patients undergoing surgery for contrast-enhancing gliomas with intra-operative MRI had a significantly higher rate of gross total resection than those in the control group. ${ }^{2}$
Figure 6: Functional Magnetic Resonance Imaging of Motor and Pre-motor Areas

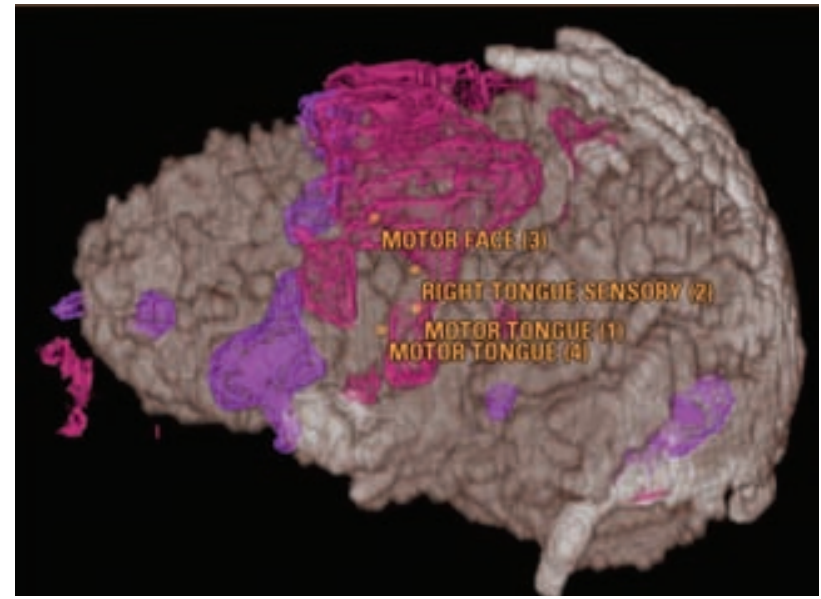

Intra-operative direct cortical mapping results are overlaid on the 3D volume. The moto region defined by functional magnetic resonance imaging (fMRI) is more extensive than that defined by direct cortical mapping; this lack of specificity makes fMRI unsuitable for the intra-operative identification of eloquent cortical regions.

Figure 8: Example Magnetoencephalograph of Somatosensory Stimuli to the Right Lip and Right Index Finger
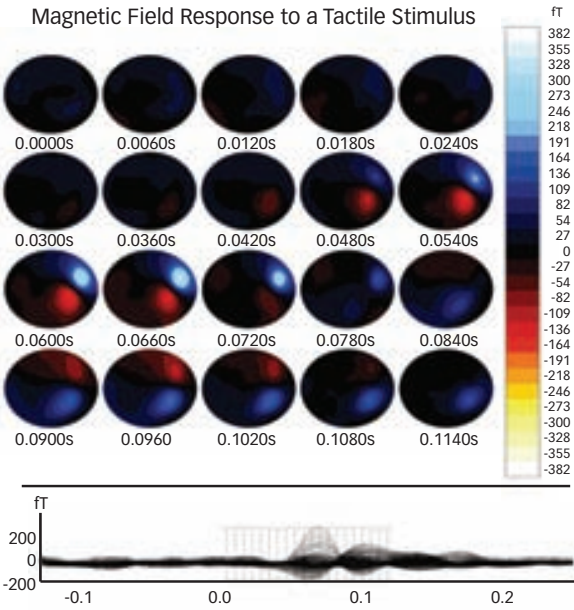

Dipole Source on MR

Multiple stimulus trials are performed for each site and cortical magnetic fields are recorded. The trials are averaged and a single dipole is reconstructed for each site using the least-square fit method. The resulting dipoles are then displayed on a co-registered T1-weighted post-gadolinium coronal magnetic resonance slice.

$M R I=$ magnetic resonance imaging; $R D 2=$ right index finger; $R L$ ip = right lip.

Despite the mounting evidence that glioma surgery with intra-operative MRI leads to better surgical outcomes, there are as yet no clear data that it leads to increased progression-free or overall survival. Although further studies will likely demonstrate these advantages, the cost of this technology is still an obstacle to its widespread adoption beyond tertiary referral centres.

\section{Intra-operative Ultrasound}

IUS is a modality that has been employed by neurosurgeons for decades. First discussed in the 1970s, IUS has been used intracranially for tumour localisation, tumour biopsy, cyst drainage and navigational guidance of ventriculo-peritoneal shunts. ${ }^{24}$ The advantages are many: IUS, like MRI, uses no ionising radiation; unlike MRI, ultrasound units are relatively inexpensive, portable and quick to use; finally, IUS can be used by the surgeon directly without the need for additional personnel (see Figure 4). 
Figure 9: Example Case of a 46-year-old Right-handed Woman with a Left Fronto-temporo-insular Low-grade Glioma

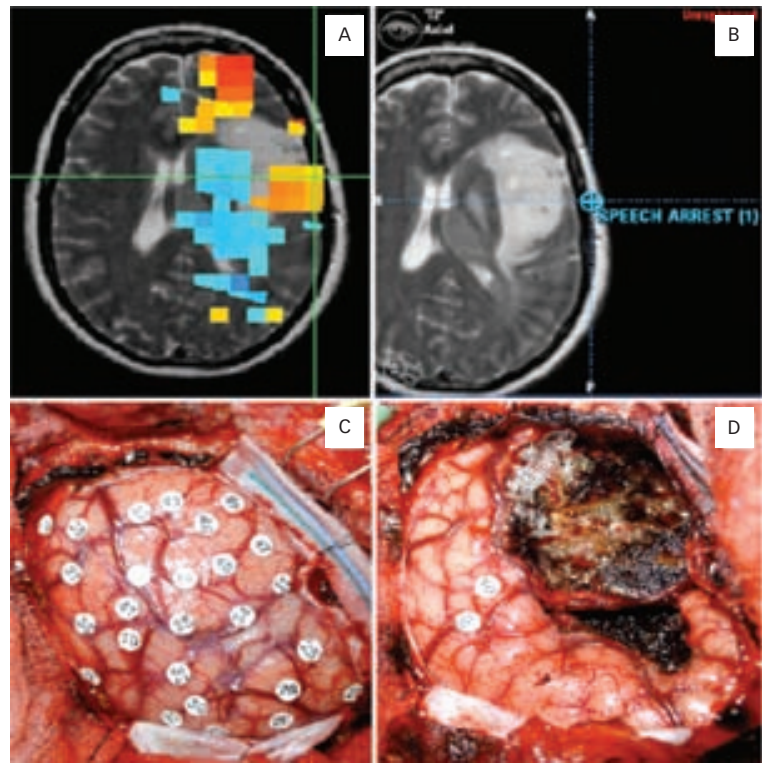

A: Magnetoencephalography analysis showing increased cortical connectivity values posterior to the tumour in the posterior frontal operculum (indicated by the orange and yellow regions marked by the green cross). B: Intra-operative neuronavigation image indicating the area where speech arrest was elicited during direct cortical mapping, marked by the blue cross. Comparison of images $A$ and $B$ revealed good correlation between the area of speech arrest and an area of increased cortical connectivity. C: Intra-operative photograph taken before tumour resection; \#9 and \#10 mark the area of speech arrest. D: Intra-operative picture after tumour resection. The temporal and insular components of the tumour have been removed; the functional language points identified (marked with the numbers 9 and 10) have been preserved.

In the last decade, IUS techniques have become more suited to neurosurgical procedures. ${ }^{25}$ In particular, 3D ultrasound is increasingly able to localise lesions within the brain parenchyma. ${ }^{26,27}$ Because IUS has the ability to resolve small anatomic structures, such as the small vessels on the cortical surface, it can be used to achieve highly accurate co-registration in a frameless stereotactical neuronavigation system. ${ }^{26}$ Similarly, IUS can be used for intra-operative re-registration of an existing MRI to account for brain shift. ${ }^{27}$ Despite these advantages, there are limitations to this technique. IUS is not optimal for deep lesions because of their distance from the probe. Additionally, it does not allow for as much tissue differentiation as other modalities, such as intra-operative MRI. Finally, IUS requires experience on the part of the user to be able to take and interpret IUS images successfully.

\section{5-Aminolevulinic Acid}

5-ALA is a small-molecule precursor that causes certain types of cancerous cells to synthesise or accumulate fluorescent porphyrins. ${ }^{28-31}$ One such porphyrin, protoporphyrin IX (PPIX), specifically accumulates in glioma cells. PPIX, when illuminated with a violet-blue $(375-440 \mathrm{~nm})$ light, emits a red fluorescence which can be viewed through a $455 \mathrm{~nm}$ long-pass filter. In effect, it makes glioma cells directly visible under the operating microscope (see Figure 5). 5-ALA is administered as an oral solution from six to 24 hours prior to surgery. Intra-operatively, the tumour is resected using standard techniques. As the pseudo-margins of the tumour are approached, fluorescence within cells may be used as one indicator that further resection is warranted.

Thus far, results with 5-ALA have been promising. It improves the extent of resection. ${ }^{32,33}$ In one study, it increased the rate of complete

\section{Figure 10: Motor Map of the Precentral Gyrus Generated with Transcranial Magnetic Stimulation}

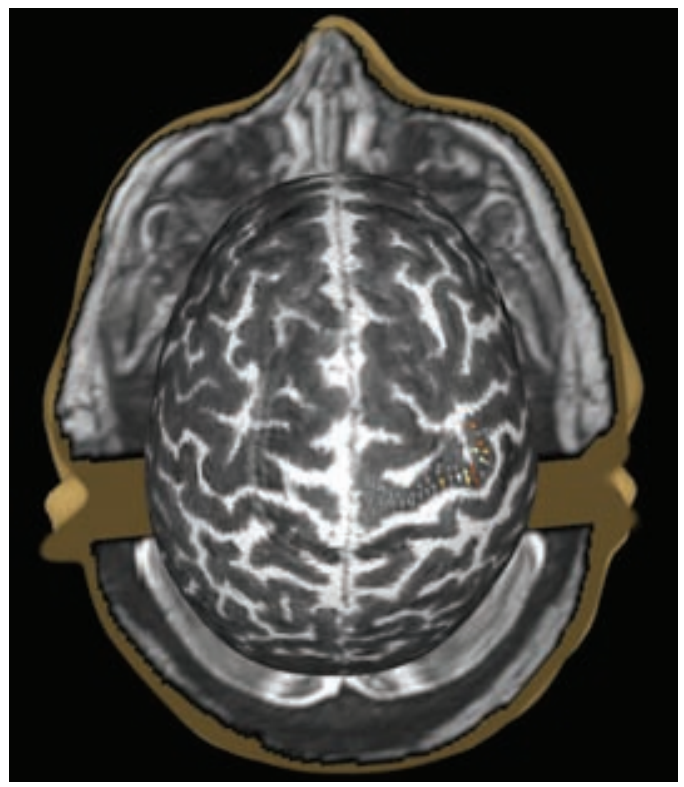

Variably coloured pins indicate the amplitude of motor-evoked potentials in the abductor pollicis brevis during the mapping procedure.

resection of the enhancing portion of glioblastoma multiforme from 36 to $65 \%$; the 5-ALA patients also doubled their progression-free survival. ${ }^{34}$ In another study, patients receiving 5-ALA had median residual tumour volumes of $0 \mathrm{~cm}$ compared with $0.5 \mathrm{~cm}$ in the conventional group. ${ }^{35}$ In that study, however, patients receiving 5-ALA were more likely to have short-term neurological deficits than control patients. This increased temporary morbidity is likely a result of the extended resections: 5-ALA does not differentiate between eloquent and non-eloquent tissue, so the resection must not be extended to the limits of fluorescence without consideration of functional pathways. Again, in this trial patients with complete resections had longer survival and time to neurological progression.

\section{Pre-operative Imaging - Integration with Intra-operative Navigation}

since its advent in 1991, fMRI has become the dominant technique for functional brain imaging. It uses the blood-oxygen-level dependence (BOLD) signal, which is a reflection of the changing levels of oxyhaemoglobin and deoxyhaemoglobin in functionally active brain regions. fMRI is limited by relatively poor temporal resolution (several seconds). It is a safe, non-invasive method that allows for whole-brain coverage, including the ability to examine activity in deep structures. Importantly, the widespread availability of MR scanners has made the technique easy to adopt across the medical field. In patients with brain tumours, fMRI has been used to identify sensorimotorcortex (see Figure 6), but other modalities such as DTI and TMS (see below) are proving to be more accurate for this purpose. Currently, the use of $f M R I$ to delineate regions associated with specific language tasks (i.e. word repetition, word reading, and object naming) is in the experimental stage. Similarly, language lateralisation with fMRI is a subject of continuing research and is rapidly reaching equivalent sensitivity and specificity to Wada testing. ${ }^{36}$

DTI is another MR-based technique used to measure the diffusion of water molecules in tissue. Because water molecules tend to diffuse preferentially along densely myelinated white-matter tracts, DTI can 
accurately trace white-matter tracts, a process known as tractography. In this modality, regions of interest that are presumed to be functionally connected are selected (i.e. primary motor cortex and cerebral peduncle). The algorithm then calculates the most likely pathway connecting those regions (see Figure 7). DTI can be performed on most modern MRI scanners. DTI-based tractography is commonly used to define the pyramidal tract ${ }^{10,37-39}$ and, to a lesser extent, language ${ }^{40}$ and visual pathways ${ }^{41,42}$ in patients with lesions in proximity to eloquent cortical and subcortical areas.

MEG measures tiny magnetic fields outside of the head that are generated by neural activity. Because it measures these fields directly, MEG offers excellent temporal resolution ( $<1 \mathrm{~ms}$ ). Furthermore, magnetic fields are unimpeded by biological tissue, so MEG recordings offer an undistorted signature of underlying neural activity. MEG scanners, however, are expensive and relatively rare, so MEG is less widespread than MR-based techniques. MEG studies are useful for localisation of sensory, motor ${ }^{43}$ (see Figure 8) and language regions (see Figure 9). ${ }^{44}$ They are also used to localise seizure foci, which is often helpful in the management of epileptogenic tumours such as oligodendrogliomas.

TMS is a technique that uses an externally applied, highly focused, brief magnetic pulse that is of sufficient strength to induce a depolarisation in the underlying neuronal units. Depending on the frequency and location of the pulse, it can either cause neuronal excitability or have a temporary lesion effect. Navigated TMS, in which the magnetic pulses are guided by a co-registered, pre-operative MRI scan, allows for highly accurate mapping of the motor system (see Figure 10). TMS is frequently used in the management of peri-Rolandic tumours, where the pyramidal tract is at highest risk of disruption from surgical resection.

Each of these four modalities can offer valuable information in the pre-operative period, assisting in planning of the surgical approach, delineating the location of the tumour with respect to eloquent brain regions and informing pre-operative discussions with patients regarding expected risks and post-operative course. However, they are highly valuable intra-operatively as well. Each of these modalities can be co-registered to a structural MRI scan and uploaded to a frameless stereotactical intra-operative neuronavigational system. With the functional imaging data thus integrated into the intra-operative navigation, it becomes a powerful tool for identifying in real time those regions that have been pre-operatively identified with a given functional study. While these techniques lack the accuracy of direct electrocortical stimulation mapping (see Figure $9 c-d$ ), they are increasingly useful adjuncts in the operative management of gliomas.

\section{Conclusion}

Intra-operative imaging is playing an increasing role in the surgical management of gliomas. Current techniques allow the surgeon to define functional brain regions, navigate accurately during the surgical resection, identify critical structures and maximise the extent of resection while preserving function. As these modalities become increasingly refined and complex, a thorough understanding of their advantages and limitations will lead to better outcomes for patients.
1. Smith JS, Chang EF, Lamborn KR, et al., Role of extent of resection in the long-term outcome of low-grade hemispheric gliomas, IClin oncol, 2008:26:1338-45.

Claus EB, Horlacher A, Hsu L, et al., Survival rates in patients with low-grade glioma after intraoperative magnetic with low-grade glioma after intraoperative magnetic
resonance image guidance, Cancer, 2005;103:1227-33. resonance image guidance, Cancer, 2005;103:1227-33.
Ahmadi R, Dictus C, Hartmann C, et al., Long-term outcome

3. Ahmadi R, Dictus $C$, Hartmann C, et al., Long-term outcom
and survival of surgically treated supratentorial low-grade and survival of surgically treated supratentorial low-grade
glioma in adult patients, Acta Neurochir (Wien), 2009;151:1359-65.

Chaichana KL, MCGirt MJ, Laterra J, et al., Recurrence and malignant degeneration after resection of adult hemispheric low-grade gliomas, J Neurosurg, 2010;112:10-7.

5. Sanai N, Polley MY, MCDermott MW, et al., An extent of resection threshold for newly diagnosed glioblastomas, I Neurosurg, 2011:115:3-8.

6. Warnke PC, Stereotactic volumetric resection of gliomas, Acta Neurochir Suppl, 2003;88:5-8

7. Krishnan R, Raabe A, Hattingen E, et al., Functional magnetic resonance imaging-integrated neuronavigation: correlatio between lesion-to-motor cortex distance and outcome, Neurosurgery, 2004;55:904-14; discussion 914-5.

8. Kurimoto $\mathrm{M}$, Hayashi $\mathrm{N}$, Kamiyama $\mathrm{H}$, et al., Impact of neuronavigation and image-guided extensive resection for neuronavigation and in single-institution retrospective study. Minim Invasive Neurosurg 2004:47:278-83.

9. Reithmeier T, Krammer M, Gumprecht $\mathrm{H}$, et al. Neuronavigation combined with electrophysiological monitoring for surgery of lesions in eloquent brain areas in 42 cases: a retrospective comparison of the neurological outcome and the quality of resection with a control group with similar lesions, Minim Invasive Neurosurg, 2003:46:65-71.

10. Wu JS, Zhou LF, Tang WJ, et al., Clinical evaluation and follow-up outcome of diffusion tensor imaging-based functional neuronavigation: a prospective, controlled study in patients with gliomas involving pyramidal tracts, Neurosurgery, 2007;61:935-48; discussion 948-39.

11. Sanai N, Berger MS, Intraoperative stimulation techniques for functional pathway preservation and glioma resection, Neurosurg Focus, 2010;28:E1

12. Black PM, Alexander E, 3rd, Martin C, et al., Craniotomy for tumor treatment in an intraoperative magnetic resonance imaging unit, Neurosurgery, 1999;45:423-31; discussion 431-23.

13. Leuthardt EC, Lim CC, Shah MN, et al., Use of movable highfield-strength intraoperative magnetic resonance imaging with awake craniotomies for resection of gliomas: with awake craniotomies for resection of gliomas: preliminary experien
discussion $205-6$.

discussion 205-6.
4. Hall WA, Liu H, Maxwell RE, Truwit CL, Influence of 1.5-Tes intraoperative MR imaging on surgical decision making, Acta Neurochir Suppl, 2003;85:29-37.

15. Nimsky C, Intraoperative MRI in glioma surgery: proof of benefit?, Lancet Oncol, 2011;12:982-3

16. Kubben PL, Ter Meulen KJ, Schijns $\mathrm{OE}$, et al., Intraoperative MRI-guided resection of glioblastoma multiforme: a systematic review, Lancet Oncol, 2011:12:1062-70.

17. Hatiboglu MA, Weinberg JS, Suki D, et al., Impact of intraoperative high-field magnetic resonance imaging guidance on glioma surgery: a prospective volumetric analysis, Neurosurgery, 2009;64:1073-81; discussion 1081.

18. Senft C, Seifert V, Hermann E, et al., Usefulness of intraoperative ultra low-field magnetic resonance imaging in glioma surgery, Neurosurgery, 2008;63(4 Suppl. 2):257-66; discussion 266-57.

19. Nimsky C, Fujita A, Ganslandt O, et al., Volumetric assessment of glioma removal by intraoperative high-field magnetic resonance imaging, Neurosurgery, 2004:55:358-70; discussion 370-1.

20. Pamir MN, Ozduman K, Dincer A, et al., First intraoperative, shared-resource, ultrahigh-field 3-Tesla magnetic resonance imaging system and its application in low-grade glioma resection, I Neurosurg, 2010;112:57-69.

21. Hirschberg H, Samset E, Hol PK, et al., Impact of intraoperative MRI on the surgical results for high-grade gliomas, Minim Invasive Neurosurg, 2005;48:77-84.

22. Senft C, Bink A, Heckelmann M, et al., Glioma extent of resection and ultra-low-field iMRI: interim analysis of a prospective randomized trial, Acta Neurochir Suppl, 2011:109:49-53.

23. Senft C, Bink A, Franz K, et al., Intraoperative MRI guidance and extent of resection in glioma surgery: a randomised, controlled trial, Lancet Oncol, 2011;12:997-1003.

24. Rubin JM, Dohrmann GJ, Use of ultrasonically guided probes and catheters in neurosurgery, Surg Neurol, 1982;18:143-8.

25. Unsgaard G, Rygh OM, Selbekk T, et al., Intra-operative 3D ultrasound in neurosurgery, Acta Neurochir (Wien), 2006;148:235-53; discussion 253

26. Nikas DC, Hartov A, Lunn K, et al., Coregistered intraoperative ultrasonography in resection of malignant glioma, Neurosurg Focus, 2003:14:e6.

27. Coenen VA, Krings T, Weidemann J, et al, Sequential visualization of brain and fiber tract deformation during intracranial surgery with three-dimensional ultrasound: an approach to evaluate the effect of brain shift, Neurosurgery, 2005;56(1 Suppl.):133-41; discussion 133-41.

28. Stummer W, Stocker S, Wagner S, et al., Intraoperative detection of malignant gliomas by 5 -aminolevulinic acid-induced porphyrin fluorescence Neurosurgery, 1998:42:518-25: discussion 525-6

29. Kriegmair $M$, Baumgartner $R$, Knuchel $R$, et al., Detection of early bladder cancer by 5-aminolevulinic acid induced porphyri fluorescence, J Urol, 1996:155:105-9: discussion 109-10.

30. Stummer W, Stocker S, Novotny A, et al., In vitro and in vivo porphyrin accumulation by $\mathrm{C} 6$ glioma cells after exposure to 5-aminolevulinic acid, J Photochem Photobiol B, 1998;45:160-9.

31. Fritsch C, Becker-Wegerich PM, Schulte KW, et al., [Photodynamic therapy and breast-plasty of a extensive superficial trunk skin basalioma of the breast. An effective combination therapy with photodynamic diagnosis] Hautarzt, 1996:47:438-42. Article in German.

32. Stummer W, Novotny A, Stepp H, et al., Fluorescence-guided resection of glioblastoma multiforme by using 5 aminolevulinic acid-induced porphyrins: a prospective study in 52 consecutive patients, J Neurosurg, 2000;93:1003-13.

33. Stummer W, Pichlmeier U, Meinel T, et al., Fluorescenceguided surgery with 5-aminolevulinic acid for resection of malignant glioma: a randomised controlled multicentre phase III trial, Lancet Oncol, 2006;7:392-401.

34. Stepp H, Beck T, Pongratz T, et al., ALA and malignant glioma: fluorescence-guided resection and photodynamic glioma: fluorescence-guided resection and photodynamic

35. Stummer W, Tonn JC, Mehdorn HM, et al., Counterbalancing risks and gains from extended resections in malignant glioma surgery: a supplemental analysis from the randomize 5-aminolevulinic acid glioma resection study. Clinical article, J Neurosurg, 2011;114:613-23.

36. Abou-Khalil B, An update on determination of language dominance in screening for epilepsy surgery: the Wada test and newer noninvasive alternatives, Epilepsia, 2007:48:442-55.

37. Berman JI, Berger MS, Chung SW, et al., Accuracy of diffusion tensor magnetic resonance imaging tractography assessed using intraoperative subcortical stimulation mapping and using intraoperative subcortical stimulation mapping and

38. Berman II, Berger MS, Mukherjee P, Henry RG, Diffusiontensor imaging-guided tracking of fibers of the pyramida tract combined with intraoperative cortical stimulation mapping in patients with gliomas, J Neurosurg, 2004;101:66-72.

39. Talos IF, Zou KH, Kikinis R, Jolesz FA, Volumetric assessment of tumor infiltration of adjacent white matter based on anatomic MRI and diffusion tensor tractography, Acad Radiol, 2007;14:431-6.

40. Henry RG, Berman Il, Nagarajan SS, et al., Subcortical pathways serving cortical language sites: initial experience with diffusion tensor imaging fiber tracking combined with intraoperative language mapping, Neuroimage, 2004;21:616-22.

41. YU CS, Li KC, Xuan Y, et al., Diffusion tensor tractography in patients with cerebral tumors: a helpful technique for neurosurgical planning and postoperative assessment, Eur J Radiol, 2005;56:197-204.

42. Werring DJ, Clark CA, Parker GJ, et al., A direct demonstration of both structure and function in the visual system: combining diffusion tensor imaging with functional magnetic resonance imaging, Neuroimage, 1999:9:352-61.

Nagarajan S, Kirsch $\mathrm{H}$, Lin P, et al., Preoperative localization Nagarajan S, Kirsch H, Lin P, et al., Preoperative localizat
of hand motor cortex by adaptive spatial filtering of magnetoencephalography data, J Neurosurg, 2008;109:228-37.

44. Edwards E, Nagarajan SS, Dalal SS, et al., Spatiotemporal imaging of cortical activation during verb generation and picture naming, Neuroimage, 2010;50:291-301. 


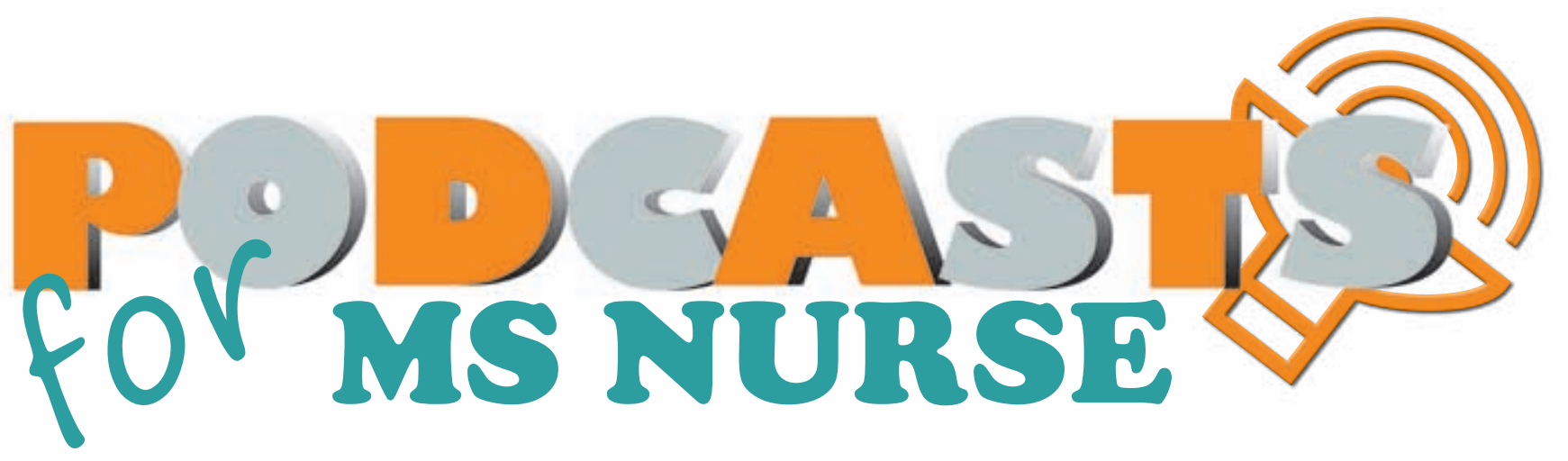

\section{available at www.iomsn.org}

New and Emerging Therapies: Challenges for MS Nursing When the Patient is Experiencing Breakthrough Disease Resources for the MS Nurse

Time Management for the MS Nurse Motivational Interviewing for the MS Nurse When the Patient is Non-adherent - Strategies for the MS Nurse Cognitive Impairment in MS - What is it and What Can the MS Nurse Do?

How can Rehabilitation Help in MS?

Fatigue in Multiple Sclerosis Depression in Multiple Sclerosis Advocacy in Multiple Sclerosis Avoiding Burnout in MS Nurses

\section{International Organization of MS Nurses MISSION STATEMENT}

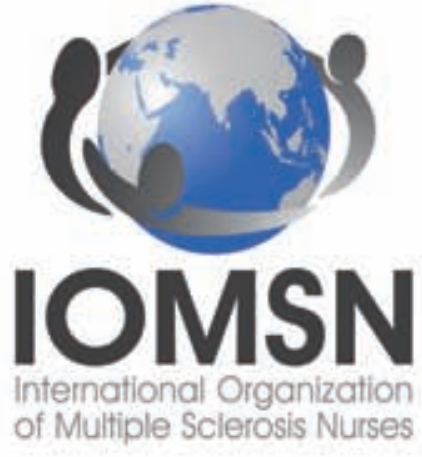

The mission of the 1OMSN is the establishment and perpetuation of a specialized branch of nursing in multiple sclerosis; to establish standards of nursing care in multiple sclerosis; to support multiple sclerosis nursing research; and to educate the health care community about multiple sclerosis; and to disseminate this knowledge throughout the world.

The ultimate goal of the IOMSN is to improve the lives of all those persons affected by multiple sclerosis through the provision of appropriate healthcare services and to make hope happen! 


\section{working to change}

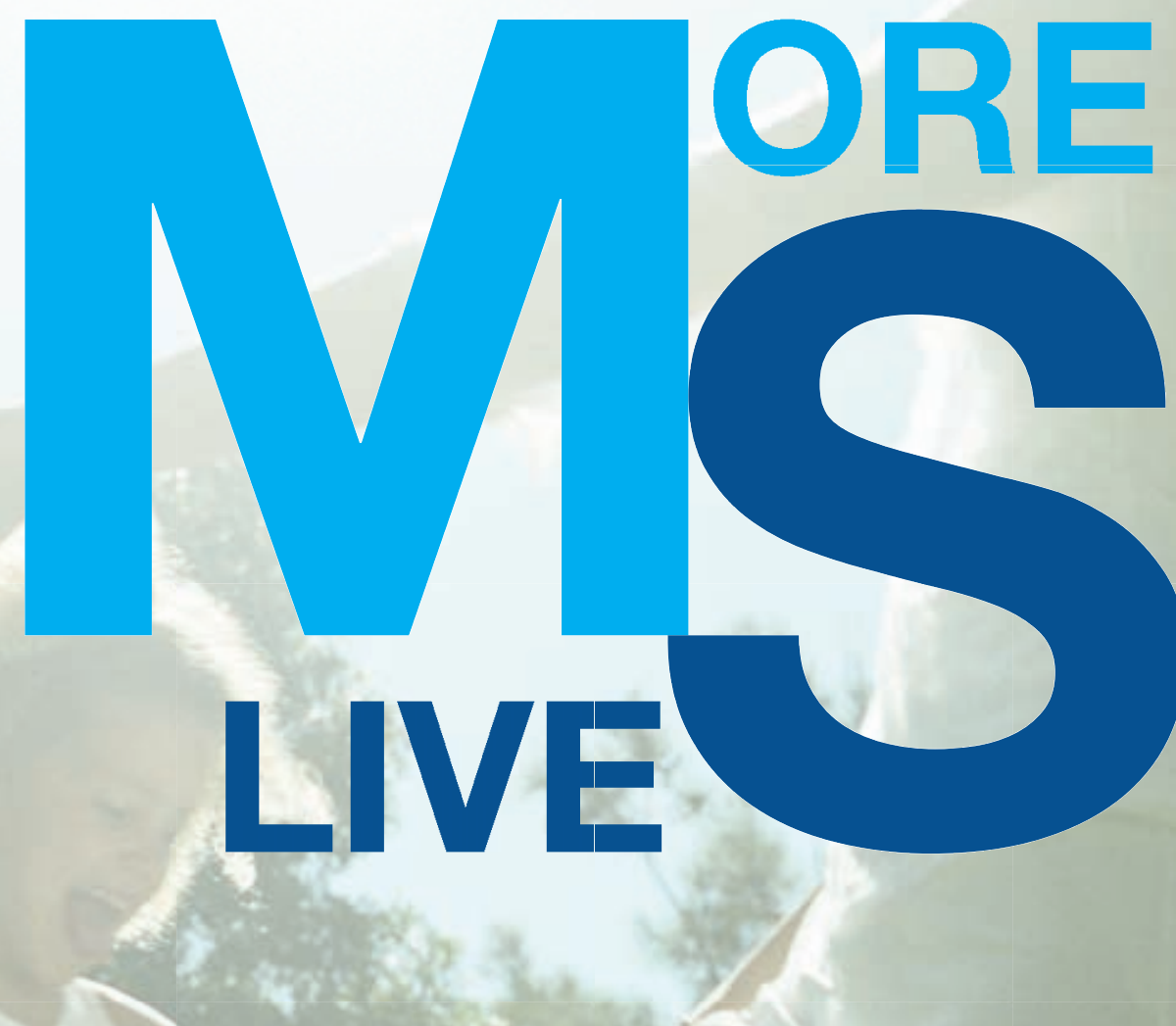

Revolutionizing the management of multiple sclerosis for patients and the ones who care for them through innovations in science. That's our commitment to the MS community.

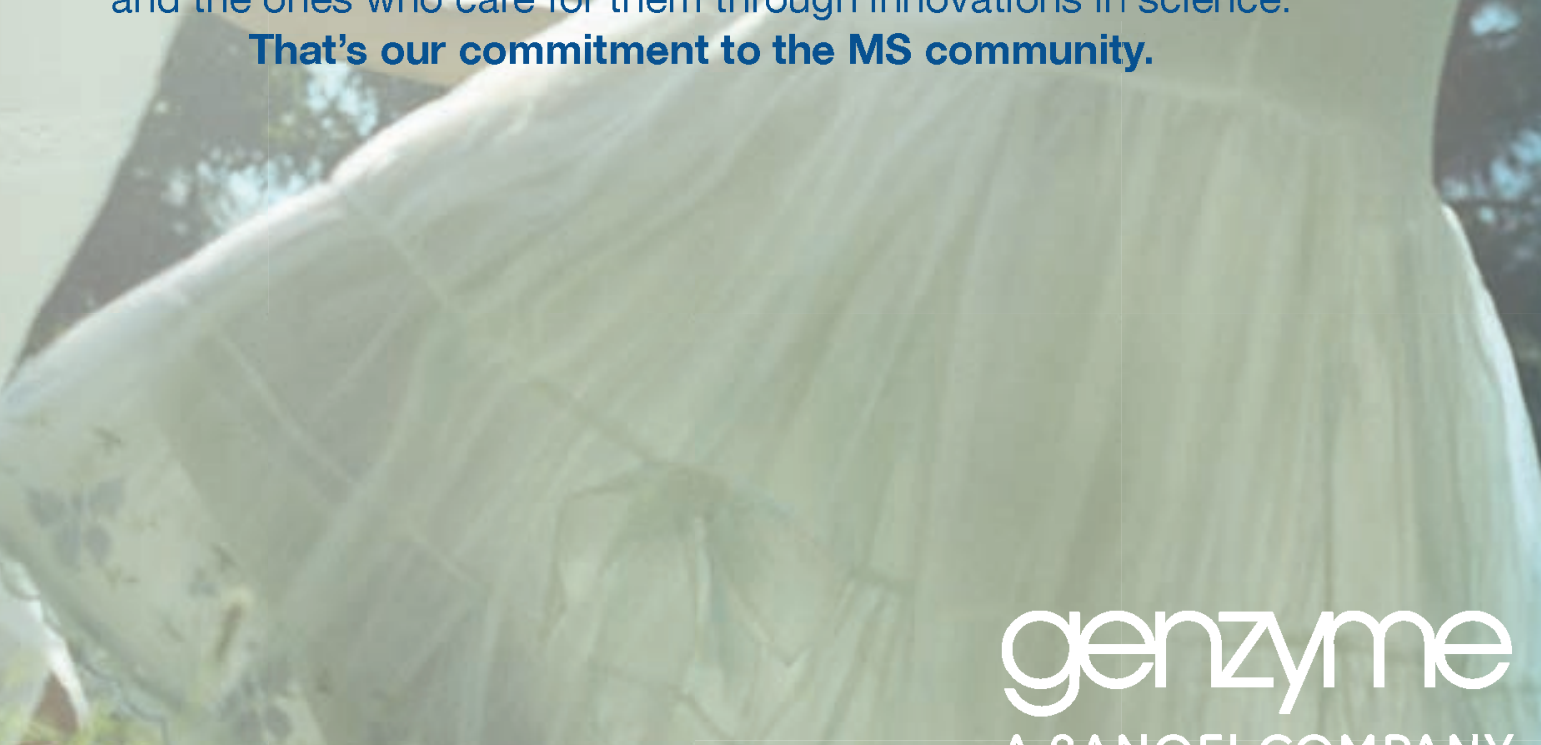

